\title{
Frugivoria por morcegos filostomídeos (Chiroptera, Phyllostomidae) em dois remanescentes urbanos de cerrado em Campo Grande, Mato Grosso do Sul
}

\author{
Jaire M. Torres ${ }^{1}$ (D), Elaine A. C. dos Anjos El, $^{1,2}$ \& Cláudia M. M. Ferreira ${ }^{3}$ (D)
}

\author{
1. Universidade Católica Dom Bosco, Av. Tamandaré, 6000, 79117-700 Campo Grande, MS, Brasil. (jairemarinho@gmail.com). \\ 2. University of Manitoba, Winnipeg, Canada. Bolsista CAPES. \\ 3. Universidade Federal de Mato Grosso do Sul, CP 549, 79070-900 Campo Grande, MS, Brasil.
}

Received 2 February 2017

Accepted 31 January 2018

Published 26 March 2018

DOI: 10.1590/1678-4766e2018002

\begin{abstract}
Frugivory by phyllostomid bats (Chiroptera, Phyllostomidae) in two cerrado urban remnants in Campo Grande, Mato Grosso do Sul. Phyllostomid bats are potential seed dispersers, due to their foraging habit and great mobility, and are the main species responsible for regeneration of neotropical forests. In Mato Grosso do Sul, research on bats diets is concentrated in the Pantanal region, with few studies focusing on the Cerrado portion, and only one study from an urban area. The objective of this study was to analyze the diets of frugivorous phyllostomid bats from two urban remnants of Cerrado in Campo Grande, Mato Grosso do Sul, evaluating whether the diet formulation of the species is based on preferences food. Bats were captured from two conservation units (the Dahma Ecological Station and Prosa State Park) for 12 nights in each area, using six mist nets, which were left open for six hours after sunset. After capture, each bat was kept in an individual cotton bag for one hour to collect a fecal sample. Fecal samples were individually stored in hermetic bottles, placed in glycerin, and then analyzed in the laboratory. All seeds found in the samples were identified. 250 bats were captured, distributed in ten species, eight genera, and two families. Phyllostomids constituted $93.2 \%$ of the captures ( $\mathrm{n}=233$ ). The most frequent species were Carollia perspicillata (Linnaeus, 1758) (27.6\%) and Artibeus lituratus (Olfers, 1818) (27.2\%). Seeds were found in 46 fecal samples from seven species of phyllostomid bats. Most of the seeds found were from the family Piperaceae ( $69.6 \%$ of the samples), and was a key resource consumed by almost all phyllostomid species. Frugivores help maintain conservation units, as they promote self-renewal, and frugivory is an important process for forest remnants. Since these bats exclusively consumed pioneer species, they play a key role in maintaining these urban remnants of Cerrado.
\end{abstract}

KEYWORDS. Conservation unit, diet, seed dispersion, fecal samples, Piperaceae.

RESUMO. Os morcegos filostomídeos são potenciais dispersores de sementes, devido ao seu hábito de forrageio e grande mobilidade, sendo os principais responsáveis pela regeneração de florestas neotropicais. No Mato Grosso do Sul as pesquisas com dieta de morcegos se concentram na região do Pantanal, com poucos trabalhos focados na porção de Cerrado, e somente um em área urbana. O objetivo deste estudo foi analisar a dieta de filostomídeos frugívoros em dois remanescentes urbanos de Cerrado em Campo Grande, Mato Grosso do Sul, avaliando-se se a formulação da dieta das espécies é baseada em preferências alimentares específicas ou não. Os morcegos foram capturados em duas unidades de conservação (a Estação Ecológica Dahma e o Parque Estadual do Prosa) por 12 noites em cada área, usando-se seis redes-de-neblina, que permaneceram abertas durante seis horas a partir do ocaso. Após a captura, cada morcego foi mantido em saco de algodão individual por uma hora para coleta das fezes. As amostras fecais foram armazenadas individualmente em frasco hermético e imersas em glicerina, sendo posteriormente analisadas em laboratório. Todas as sementes encontradas nas amostras foram identificadas. Foram capturados 250 morcegos, distribuídos em dez espécies, oito gêneros e duas famílias. Os filostomídeos constituíram $93,2 \%$ das capturas $(\mathrm{n}=233)$. As espécies mais frequentes foram Carollia perspicillata (Linnaeus, 1758) (27,6\%) e Artibeus lituratus (Olfers, 1818) $(27,2 \%)$. Foram encontradas sementes em 46 amostras fecais de sete espécies de morcegos filostomídeos. A maioria das sementes encontradas era da família Piperaceae (69,6\% das amostras), sendo um recurso-chave consumido por quase todas as espécies de filostomídeos. Os frugívoros auxiliam na manutenção de unidades de conservação, pois promovem a auto-renovação, sendo a frugivoria um processo importante em remanescentes florestais. $\mathrm{O}$ consumo exclusivo de espécies pioneiras é um indício do papel de morcegos na manutenção destes remanescentes urbanos de Cerrado.

PALAVRAS-CHAVE. Dieta, dispersão de sementes, Piperaceae, unidades de conservação.

Remanescentes de vegetação natural circundados por uma matriz urbana apresentam suas condições naturais quase que completamente alteradas (FEIBER, 2004), com mudanças no volume dos recursos hídricos, transformações nas bordas dos remanescentes (RARES \& BRANDIMARTE, 2014), predomínio de árvores baixas e baixa cobertura vegetal (MofFATT et al., 2004). A alteração desses ambientes geralmente resulta em significativas alterações na composição da fauna, podendo ser considerados como ambientes defaunados (Melo et al., 2011).

A defaunação resulta na alteração das interações locais e, consequentemente, na manutenção dos remanescentes 
florestais (KAGEYAMA \& GANDARA, 2003), como exemplo, a redução das populações de animais frugívoros pode comprometer populações vegetais zoocóricas (SILVA \& TABARELLI, 2000). A manutenção destes ambientes ocorre em grande parte através de auto-renovação, sendo a dispersão de sementes pela fauna um processo importante para a regeneração natural e perpetuação de povoamentos vegetais (Deminicis et al., 2009).

Os morcegos frugívoros apresentam grande importância na manutenção de ambientes naturais, participando de processos essenciais à manutenção de ambientes florestais e regeneração de áreas degradadas (FLEMING \& SosA, 1994). Uma grande parcela destes animais se alimenta de frutos de plantas pioneiras, dispersando suas sementes para longe das matrizes durante seu deslocamento, que podem ser transportadas para clareiras e áreas degradadas (GALINDOGoNZÁLEZ et al., 2000), possibilitando o processo de sucessão secundária (CHARLEs-DominiQue, 1986). Os morcegos da família Phyllostomidae, por exemplo, se distribuem por toda a Região Neotropical, sendo considerados os maiores responsáveis pela regeneração das florestas (HUTSON et al., 2001). Esta região concentra o maior número de plantas com frutos carnosos e atrativos aos animais frugívoros (WILLSON, 1991), onde o Brasil se destaca, com registro de ao menos 189 espécies de plantas utilizadas por morcegos (FABIÁN et al., 2008).

O conhecimento sobre a história natural de morcegos possibilita o reconhecimento de seus padrões (RICKLEFS, 1990), como sua riqueza, distribuição, abundância relativa e uso de recursos. Diante da possibilidade de manutenção de ambientes naturais através dos morcegos, o conhecimento aprofundado sobre o papel deles na dispersão de sementes e processos de restauração pode ter importância na recuperação de áreas degradadas e em ações de conservação (BERNARD et al., 2012). A compreensão da interação entre morcegos e plantas têm proporcionado novas perspectivas de planejamento, como a utilização de óleos essenciais para atração de morcegos e consequente aumento na chuva de sementes em locais de interesse, o que pode fortalecer processos tradicionais de recuperação que recuperam somente parte das funções do ambiente (Mikich et al., 2015). Dessa forma, o conhecimento sobre os padrões de interação em determinada região, pode ser essencial para o planejamento das medidas de restauração adequadas.

No estado do Mato Grosso do Sul, as pesquisas sobre dieta de morcegos se concentram na região do Pantanal (TeixeIra et al., 2009; OliveIra \& Lemes, 2010; Munin et al., 2012; Porfírio \& BordignON, 2015), com poucos trabalhos na porção de Cerrado (MARTINs et al., 2014a; 2014b), sendo que somente um deles aborda tais interações em fragmentos urbanos (MARTINs et al., 2014a). O objetivo deste estudo foi caracterizar a frugivoria por morcegos filostomídeos em dois remanescentes urbanos de Cerrado em Campo Grande, Mato Grosso do Sul, identificando quais espécies de plantas são os principais itens alimentares dos morcegos frugívoros capturados.

\section{MATERIAL E MÉTODOS}

Locais de estudo. As coletas foram realizadas na Estação Ecológica Dahma ( $\left.20^{\circ} 28^{\prime} \mathrm{S} 54^{\circ} 32^{\prime} \mathrm{W}\right)$ e no Parque Estadual do Prosa (20 $27^{\prime}$ 'S 54 $\left.33^{\circ} \mathrm{W}\right)$, unidades de conservação localizadas no perímetro de Campo Grande, Mato Grosso do Sul. O clima da região é Tropical Chuvoso de Savana (subtipo Aw) segundo a classificação de Köppen (Peel et al., 2007), com temperatura média de $23,3^{\circ} \mathrm{C}$ e precipitação média anual de 1579,7 mm (HONER, 1993).

Área 1 (A1): a Estação Ecológica Dahma (EED) possui área aproximada de 42,3 ha, e se situa na região da Área de Proteção Ambiental (APA) do Lajeado. A hidrografia da região é caracterizada pela presença do córrego Lajeado. A cobertura vegetal da APA é formada por fisionomias presentes no domínio dos Cerrados: cerrado sensu stricto, cerradão, mata ciliar, vereda e mata inundável. Foram amostradas porções de cerradão e mata ciliar, sendo essas as fisionomias presentes na EED.

Área 2 (A2): O Parque Estadual do Prosa (PEP), abriga as duas nascentes do córrego Prosa, os córregos Joaquim Português e Desbarrancado. Sua área é de aproximadamente $135 \mathrm{ha}$, com formações de mata de galeria, cerrado sensu stricto e cerradão, sendo realizadas amostragens em porções de todas as fisionomias encontradas no PEP.

Coleta de dados. Cada fragmento foi amostrado mensalmente de março a agosto de 2009 e de fevereiro a maio de 2010, além de duas coletas em dezembro de 2009 na EED e duas coletas em agosto de 2010 no PEP, totalizando também 12 noites de captura em cada local. Os morcegos foram capturados com auxílio de seis redes-de-neblina, utilizando-se em cada área seis redes de 12,0 x 2,5 m nas seis primeiras sessões, e três redes de 7,0 x 2,0 $\mathrm{m}$ e três de $12,0 \mathrm{x}$ $2,5 \mathrm{~m}$ nas seis coletas subsequentes. As redes permaneceram abertas por seis horas a partir do ocaso, sendo verificadas em intervalos de 20 minutos. As redes foram fixadas entre 0,5 e 3,0 $\mathrm{m}$ acima do solo, em trilhas e clareiras no interior da mata. O esforço total de captura (cf. STRAUBE \& BianCONI, 2002) empregado foi de 22.464 h.m , sendo $^{2} 1.232$ h.m ${ }^{2}$ em cada área.

Após a captura, cada morcego foi mantido em um saco de algodão individual pelo período de uma hora para obtenção das fezes, não havendo a reutilização de um saco de algodão na mesma noite. Os morcegos foram identificados de acordo com VizotTo \& TADDEI (1973) e de atualizações (REIS et al., 2007), sendo posteriormente soltos no mesmo local. Todos os procedimentos foram realizados de acordo com as licenças Sisbio n 10615-1 e 20548-2, com o depósito de espécimes-testemunho na Coleção Zoológica da Universidade Federal de Mato Grosso do Sul (ZUFMS) e no Laboratório de Zoologia dos Vertebrados da Universidade Católica Dom Bosco (LZV), respectivamente a cada licença.

Cada amostra de fezes foi acondicionada individualmente em frasco hermético de $1,5 \mathrm{ml}$ e imersa em glicerina. No laboratório as amostras fecais foram lavadas com água destilada em placas de petri, removendo-se as sementes para posterior identificação. As sementes foram 
identificadas com auxílio de microscópio estereoscópico, comparando-as com o banco de referência de sementes da Universidade Federal de Mato Grosso do Sul.

Análise de dados. Foi considerada uma amostra fecal todo o conteúdo depositado no saco de algodão por um único morcego. Quando uma mesma amostra fecal apresentou mais de uma espécie vegetal, estas foram consideradas como amostras distintas. A análise da dieta dos morcegos frugívoros foi realizada através da frequência de ocorrência dos recursos encontrados nas amostras fecais.

A diversidade da quiropterofauna local e da dieta de cada espécie de morcego foi avaliada pelo Índice de Diversidade de Shannon (Aguiar \& Marinho-Filho, 2007) com o programa BioDiversity Pro (MCALEECE et al., 1997). Foi verificada a similaridade entre as comunidades de morcegos de cada área, além da similaridade entre a dieta das espécies pelo índice de Morisita (1959), utilizando-se o programa PAST 2.10 (HAMmER et al., 2001).

Para avaliar se a dieta das espécies é baseada em suas preferências alimentares ou nos recursos disponíveis em cada fragmento, foi realizada uma análise de agrupamento baseada na frequência de ocorrência dos itens alimentares, utilizando-se o índice de MoRISITA (1959) no programa PAST 2.10 (Hammer et al., 2001). Com essa análise é possível organizar grupos com base em sua dieta, observando-se a ocorrência de dois possíveis padrões de agrupamento: 1) agrupamentos de populações da mesma espécie ou congêneres e provenientes de ambas as áreas, indicando uma dieta baseada em preferências alimentares e independente da origem das populações; ou 2) agrupamentos de populações de diferentes espécies e provenientes de uma mesma área, indicando uma dieta baseada nas condições locais e não nas possíveis preferencias específicas.

\section{RESULTADOS}

Foram capturados 250 morcegos de 11 espécies distribuídas em duas famílias, Phyllostomidae e Vespertilionidae (Tab. I). Phyllostomidae apresentou as espécies frugívoras Artibeus lituratus (Olfers, 1818), Artibeus planirostris (Spix, 1823), Carollia perspicillata (Linnaeus, 1758), Chiroderma doriae Thomas, 1891, Chiroderma villosum Peters, 1860, Platyrrhinus helleri (Thomas, 1912), Platyrrhinus lineatus (E. Geoffroy, 1810) e Sturnira lilium (E. Geoffroy, 1810) (Phyllostomidae), além da espécie nectarívora Glossophaga soricina (Pallas, 1766). Vespertilionidae foi representada por Lasiurus blossevillii (Lesson \& Garnot, 1826) e Myotis nigricans (Schinz, 1821), ambas espécies insetívoras. Os filostomídeos constituíram $93,2 \%$ das capturas $(\mathrm{n}=233)$, sendo as espécies mais frequentes as frugívoras C. perspicillata e A. lituratus, com $27,6 \%(n=69)$ e $27,2 \%(n=68)$ de frequência respectivamente.

A maior riqueza e abundância de morcegos foram verificadas na EED, com 133 registros de morcegos de dez espécies, enquanto no PEP foram realizados 117 registros de nove espécies. Embora com menor riqueza e abundância, o PEP apresentou maior diversidade de quiropterofauna $\left(H^{\prime}=0,803\right)$, uma vez que na EED $\left(H^{\prime}=0,771\right)$ houve o predomínio de $C$. perspicillata e $A$. lituratus (Tab. I). A similaridade na composição da quiropterofauna entre as duas unidades de conservação foi de $\mathrm{Cmh}=0,8803$.

Foram analisadas 46 amostras fecais de sete espécies de morcegos, onde se encontraram sementes de cinco espécies vegetais, representantes de quatro gêneros e quatro famílias. A família mais consumida foi Piperaceae, encontrada em 69,6\% das amostras, seguida por Solanaceae (13,0\%), Moraceae $(10,8 \%)$ e Urticaceae $(6,5 \%)$. Em ambas as áreas o item

Tab. I. Frequência de capturas de morcegos entre março de 2009 e maio de 2010 em duas unidades de conservação de Campo Grande, Mato Grosso do Sul, Brasil [n, número de morcegos capturados; A1, Estação Ecológica Dahma (EED); A2, Parque Estadual do Prosa (PEP); $\sum U C$, soma de capturas nas duas unidades de conservação; $\mathrm{NC}$, número de tombo dos exemplares].

\begin{tabular}{|c|c|c|c|c|}
\hline \multirow{2}{*}{ Família/espécie } & \multicolumn{2}{|c|}{$\mathrm{UC} n(\%)$} & \multirow{2}{*}{$\begin{array}{l}\sum \mathrm{UC} \\
\mathrm{n}(\%)\end{array}$} & \multirow{2}{*}{$\mathrm{NC}$} \\
\hline & A1 & A2 & & \\
\hline \multicolumn{5}{|l|}{ Phyllostomidae } \\
\hline Artibeus lituratus (Olfers, 1818) & $33(24,8)$ & $35(29,9)$ & $68(27,2)$ & LZV016 \\
\hline Artibeus planirostris (Spix, 1823) & $9(6,8)$ & $5(4,3)$ & $14(5,6)$ & \\
\hline Carollia perspicillata (Linnaeus, 1758) & $48(36,1)$ & $21(17,9)$ & $69(27,6)$ & LZV017; ZUFMS497 \\
\hline Chiroderma doriae Thomas, 1891 & $4(3,0)$ & $1(0,9)$ & $5(2,0)$ & \\
\hline Chiroderma vilosum Peters, 1860 & $1(0,8)$ & 0 & $1(0,4)$ & ZUFMS492 \\
\hline Glossophaga soricina (Pallas, 1766) & $5(3,8)$ & $8(6,8)$ & $13(5,2)$ & ZUFMS499 \\
\hline Platyrrhinus helleri (Thomas, 1912) & $2(1,5)$ & 0 & $2(0,8)$ & \\
\hline Platyrrhinus lineatus (E. Geoffroy, 1810) & $10(7,5)$ & $11(9,4)$ & $21(8,4)$ & LZV015; ZUFMS506 \\
\hline Sturnira lilium (E. Geoffroy, 1810) & $18(13,5)$ & $22(18,8)$ & $40(16,0)$ & LZV018; LZV020 \\
\hline \multicolumn{5}{|l|}{ Vespertilionidae } \\
\hline Lasiurus blossevillii (Lesson \& Garnot, 1826) & 0 & $1(0,9)$ & $1(0,4)$ & LZV014 \\
\hline Myotis nigricans (Schinz, 1821) & $3(2,3)$ & $13(11,1)$ & $16(6,4)$ & \\
\hline Capturas $\left(\sum\right)$ & 133 & 117 & 250 & \\
\hline Riqueza & 10 & 9 & 11 & \\
\hline Shannon H’ & 0,771 & 0,803 & & \\
\hline
\end{tabular}


mais consumido foi Piper tuberculatum Jacq., sendo 73,5\% dos registros de consumo na EED (Tab. II) e 58,3\% no PEP (Tab. III). A similaridade no consumo de frutos entre as duas áreas foi de $\mathrm{Cmh}=0,9600$.

Carollia perspicillata, a mais abundante, apresentou dieta composta por $95 \%$ de P. tuberculatum na EED (Tab. II), além de ser o único item consumido por essa espécie no PEP (Tab. III), resultando em $\mathrm{Cmh}=0,9974$ de similaridade na frugivoría entre as áreas (Tab. IV). Devida à baixa riqueza de itens consumidos, a diversidade da dieta de C. perspicillata foi de apenas $\mathrm{H}^{\prime}=0,086$ na EED e de $\mathrm{H}^{\prime}=0$ no PEP (Tab. II e III).

Artibeus lituratus, também abundante nas áreas, apresentou a maior diversidade do consumo de frutos dentre as espécies frugivoras capturadas, sendo de $H^{\prime}=0,602$ na EED e H'=0,577 no PEP (Tab. III), com similaridade de dieta de $\mathrm{Cmh}=0,7894$ entre as áreas (Tab. IV). Na EED, $P$. lineatus e $S$. lilium apresentaram diversidade de dieta de $\mathrm{H}^{\prime}=0,477$ e $\mathrm{H}^{\prime}=0,301$ respectivamente, e $\mathrm{H}^{\prime}=0$ no $\mathrm{PEP}$ para ambas (Tabs II, III). Não foi possível avaliar a diversidade da dieta de A. planirostris, G. soricina e P. helleri, devido à quantidade reduzida de amostras obtidas, enquanto $C$. doriae e $C$. vilosum não forneceram amostras fecais.

AAnálise de Agrupamento demonstrou dois grupos, cada um composto por populações de ambos os fragmentos, indicando a utilização de recursos baseada em preferências de cada espécie e não nos itens alimentares de cada fragmento. Os grupos formados foram: Grupo 1) Consumidores de $P$. tuberculatum: reuniu populações de $A$. planirostris e $P$. helleri da EED, S. lilium do PEP e C. perspicillata de ambas as áreas, apresentando similaridade mínima de $\mathrm{Cmh}=0,9974$; e Grupo 2) Consumidores de Solanum sp.: reuniu a população de $S$. lilium da EED e as populações de $A$. lituratus de ambas as áreas, com similaridade mínima de $\mathrm{Cmh}=0,6667$ (Fig. 1).

\section{DISCUSSÃO}

As espécies mais abundantes nos fragmentos estudados - C. perspicillata, A. lituratus, S. lilium e $P$. lineatus - são comuns em áreas urbanas no Brasil (BREDT \& UiEDA, 1996; LimA, 2008). O predomínio de filostomídeos em levantamentos com redes-de-neblina é um fato comum, além de esta família ser a mais rica na região Neotropical (FENTON et al., 1992). A ocorrência de morcegos frugívoros é um importante fator para a manutenção de áreas florestais, podendo auxiliar no estabelecimento de organismos vegetais por dispersão de sementes e por consequência na autorenovação destes ambientes (LE BouRLEGAT, 2003).

Carollia perspicillata pode ser considerada de alto potencial na manutenção dos fragmentos, pois morcegos desse gênero são considerados especialistas no consumo

Tab. II. Frequência de itens consumidos e diversidade da dieta de morcegos filostomídeos entre março de 2009 e maio de 2010 na Estação Ecológica Dahma (A1), em Campo Grande, Mato Grosso do Sul, Brasil (Al, Artibeus lituratus; Ap, Artibeus planirostris; Cp, Carollia perspicillata; Gs, Glossophaga soricina; $\mathrm{Ph}$, Platyrrhinus helleri; Pl, Platyrrhinus lineatus; S1, Sturnira lilium; $\sum$, Soma das amostras da área amostrada).

\begin{tabular}{|c|c|c|c|c|c|c|c|c|c|}
\hline Item alimentar & Al n $(\%)$ & $\begin{array}{c}\text { Ap } \\
n(\%)\end{array}$ & $\mathrm{Cp} \mathrm{n}(\%)$ & Gs $n(\%)$ & $\begin{array}{c}\mathrm{Ph} \\
\mathrm{n}(\%)\end{array}$ & & $\begin{array}{c}\mathrm{Pl} \\
\mathrm{n}(\%)\end{array}$ & $\begin{array}{c}\mathrm{S} 1 \\
\mathrm{n}(\%)\end{array}$ & $\sum_{\mathrm{n}(\%)}$ \\
\hline \multicolumn{10}{|l|}{ Moraceae } \\
\hline Ficus crocata (Miq.) & $1(25,0)$ & 0 & 0 & 0 & 0 & 1 & $(33,3)$ & 0 & $2(5,9)$ \\
\hline Ficus insipida Willd. & $1(25,0)$ & 0 & 0 & 0 & 0 & & 0 & 0 & $1(2,9)$ \\
\hline \multicolumn{10}{|l|}{ Piperaceae } \\
\hline Piper tuberculatum Jacq. & $1(25,0)$ & $1(100,0)$ & $19(95,0)$ & 0 & $1(100,0)$ & 1 & $(33,3)$ & $2(50,0)$ & $25(73,5)$ \\
\hline \multicolumn{10}{|l|}{ Solanaceae } \\
\hline Solanum sp. & $1(25,0)$ & 0 & $1(5,00)$ & 0 & 0 & & 0 & $2(50,0)$ & $4(11,8)$ \\
\hline \multicolumn{10}{|l|}{ Urticaceae } \\
\hline Cecropia pachystachya Trécul & 0 & 0 & 0 & 0 & 0 & 1 & $(33,3)$ & 0 & $2(5,9)$ \\
\hline Total de Amostras & 4 & 1 & 20 & 0 & 1 & & 3 & 4 & 34 \\
\hline Total de itens & 4 & 1 & 2 & 0 & 1 & & 3 & 2 & 5 \\
\hline Shannon H' & 0,602 & 0 & 0,086 & 0 & 0 & & 0,477 & 0,301 & 0,397 \\
\hline
\end{tabular}

Tab. III. Frequência de itens consumidos e diversidade da dieta de morcegos filostomídeos entre março de 2009 e maio de 2010 no Parque Estadual do Prosa (A2), em Campo Grande, Mato Grosso do Sul, Brasil (A1, Artibeus lituratus; Ap, Artibeus planirostris; Cp, Carollia perspicillata; Gs, Glossophaga soricina; $\mathrm{Ph}$, Platyrrhinus helleri; $\mathrm{Pl}$, Platyrrhinus lineatus; $\mathrm{S1}$, Sturnira lilium; $\sum$, soma das amostras da área amostrada).

\begin{tabular}{|c|c|c|c|c|c|c|c|c|}
\hline Item alimentar & Al n(\%) & Ap n $(\%)$ & $\mathrm{Cp} \mathrm{n}(\%)$ & Gs n(\%) & $\mathrm{Ph} \mathrm{n}(\%)$ & $\mathrm{Pl} n(\%)$ & Sl n $(\%)$ & $\sum \mathrm{n}(\%)$ \\
\hline \multicolumn{9}{|l|}{ Moraceae } \\
\hline Ficus insipida & $1(16,7)$ & 0 & 0 & $1(100,0)$ & 0 & 0 & 0 & $2(16,7)$ \\
\hline \multicolumn{9}{|l|}{ Piperaceae } \\
\hline Piper tuberculatum & $2(33,3)$ & 0 & $4(100,0)$ & 0 & 0 & 0 & $1(100,0)$ & $7(58,3)$ \\
\hline \multicolumn{9}{|l|}{ Solanaceae } \\
\hline Solanum sp. & $2(33,3)$ & 0 & 0 & 0 & 0 & 0 & 0 & $2(16,7)$ \\
\hline \multicolumn{9}{|l|}{ Urticaceae } \\
\hline Cecropia pachystachya & $1(16,7)$ & 0 & 0 & 0 & 0 & 0 & 0 & $1(8,3)$ \\
\hline Total de Amostras & 6 & 0 & 4 & 1 & 0 & 0 & 1 & 12 \\
\hline Total de itens & 4 & 0 & 1 & 1 & 0 & 0 & 1 & 4 \\
\hline Shannon H' & 0,577 & 0 & 0 & 0 & 0 & 0 & 0 & 0,486 \\
\hline
\end{tabular}


Tab. IV. Similaridade da dieta de filostomídeos frugívoros capturados entre março de 2009 e maio de 2010 em duas unidades de conservação de Campo Grande, Mato Grosso do Sul, Brasil [Al, Artibeus lituratus; Ap, Artibeus planirostris; Cp, Carollia perspicillata; Gs, Glossophaga soricina; $\mathrm{Ph}$, Platyrrhinus helleri; Pl, Platyrrhinus lineatus; S1, Sturnira lilium; A1, Estação Ecológica Dahma (EED); A2, Parque Estadual do Prosa (PEP)].

\begin{tabular}{|c|c|c|c|c|c|c|c|c|c|c|}
\hline & $\begin{array}{c}\mathrm{Al} \\
\text { (A1) }\end{array}$ & $\begin{array}{c}\mathrm{A} 1 \\
\text { (A2) }\end{array}$ & $\begin{array}{c}\text { Ap } \\
\text { (A1) }\end{array}$ & $\mathrm{Cp}(\mathrm{A} 1)$ & $\mathrm{Cp}(\mathrm{A} 2)$ & Gs (A2) & $\mathrm{Ph}(\mathrm{A} 1)$ & Pl (A1) & $\mathrm{Sl}$ (A1) & $\mathrm{Sl}(\mathrm{A} 2)$ \\
\hline $\begin{array}{c}\mathrm{Al} \\
\text { (A1) }\end{array}$ & 1 & 0.7895 & 0.4000 & 0.4329 & 0.4 & 0.4000 & 0.4000 & 0.5714 & 0.6667 & 0.4000 \\
\hline $\begin{array}{c}\mathrm{A} 1 \\
\text { (A2) }\end{array}$ & 0.7894 & 1 & 0.5217 & 0.5636 & 0.5217 & 0.2609 & 0.5217 & 0.7273 & 0.8571 & 0.5217 \\
\hline $\begin{array}{c}\text { Ap } \\
\text { (A1) }\end{array}$ & 0.4000 & 0.5217 & 1 & 0.9974 & 1 & 0 & 1 & 0.5000 & 0.6667 & 1 \\
\hline $\begin{array}{c}\mathrm{Cp} \\
\text { (A1) }\end{array}$ & 0.4329 & 0.5636 & 0.9974 & 1 & 0.9974 & 0 & 0.9974 & 0.5114 & 0.7117 & 0.9974 \\
\hline $\begin{array}{c}\mathrm{Cp} \\
\text { (A2) }\end{array}$ & 0.4000 & 0.5217 & 1 & 0.9974 & 1 & 0 & 1 & 0.5000 & 0.6667 & 1 \\
\hline $\begin{array}{c}\text { Gs } \\
\text { (A2) }\end{array}$ & 0.4000 & 0.2609 & 0 & 0 & 0 & 1 & 0 & 0.5000 & 0 & 0 \\
\hline $\begin{array}{c}\mathrm{Ph} \\
\text { (A1) }\end{array}$ & 0.4000 & 0.5217 & 1 & 0.9974 & 1 & 0 & 1 & 0.5000 & 0.6667 & 1 \\
\hline $\begin{array}{c}\mathrm{Pl} \\
\text { (A1) }\end{array}$ & 0.5714 & 0.7272 & 0.5 & 0.5114 & 0.5000 & 0.5000 & 0.5000 & 1 & 0.4000 & 0.5000 \\
\hline $\begin{array}{c}\text { S1 } \\
\text { (A1) }\end{array}$ & 0.6667 & 0.8571 & 0.6667 & 0.7117 & 0.6667 & 0 & 0.6667 & 0.4000 & 1 & 0.6667 \\
\hline $\begin{array}{c}\mathrm{S} 1 \\
\text { (A2) }\end{array}$ & 0.4000 & 0.5217 & 1 & 0.9974 & 1 & 0 & 1 & 0.5000 & 0.6667 & 1 \\
\hline
\end{tabular}

(BIZERRIL \& RAW, 1997) e os maiores dispersores de Piper spp. (Hutson et al., 2001), sendo as plantas desse gênero pertencentes a um grupo-chave para o processo de nucleação e que proporciona a agregação de outras espécies ao seu redor (Miкich et al., 2015). Essa relação é determinada em grande parte pelo predomínio de espécies de Piper na alimentação de Carollia spp. na Região Neotropical (AguiRre et al., 2003; GianNini \& KalKo, 2004), ressaltado também no Brasil em diversos estudos (e.g. PINTO \& ORTÊNCIO-FILHO, 2006; Aguiar \& Marinho-Filho, 2007; SATO et al., 2008; Brito et al., 2010; Munin et al., 2012). Embora o predomínio de $P$. tuberculatum já seja relatado na dieta de C.perspicillata em remanescentes urbanos de Campo Grande (MARTins et al., 2014b), o registro de Solanum sp. demonstra uma interação já conhecida (AGUIAR \& MARINHO-FILHO, 2007), mas ainda não relatada na região urbana do Mato Grosso do Sul.

Artibeus lituratus é predominantemente frugívora (ZorTÉa, 2007) e conhecida pelo consumo de frutos de Moraceae e Urticaceae (PAssos et al., 2003; PAssos \& Graciolli, 2004). Apesar de serem indicadas preferências por alguns itens, pode apresentar uma dieta generalista em locais ou épocas de baixa densidade de recursos, demonstrando plasticidade alimentar em resposta à variação na disponibilidade de alimentos (PASSOS \& GRACIOLLI, 2004; BRUSCO \& TOZATO, 2009). Esta capacidade foi corroborada no presente estudo pela variedade de recursos consumidos pela espécie, apresentando a maior diversidade de dieta quando comparada com outras espécies em ambas as áreas.

Somente uma amostra fecal de A. planirostris foi obtida, ressaltando a presença de poucas amostras coletadas para essa espécie também em outros estudos de frugivoria (e.g. PAssos et al., 2003; Brito et al., 2010; Martins et al.,

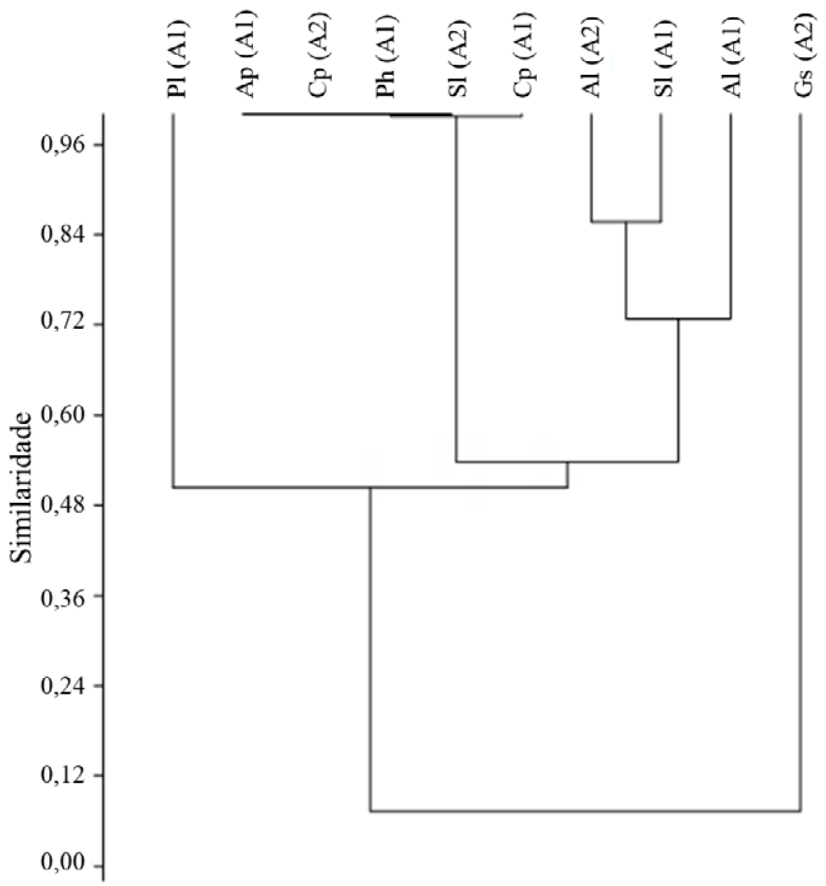

Fig. 1. Análise de Agrupamento através do Índice de Similaridade de Morisita entre a dieta das espécies de morcegos frugívoros capturados entre março de 2009 e maio de 2010 em duas unidades de conservação de Campo Grande, Mato Grosso do Sul, Brasil [Al, Artibeus lituratus; Ap, Artibeus planirostris; $\mathrm{Cp}$, Carollia perspicillata; Gs, Glossophaga soricina; $\mathrm{Ph}$, Platyrrhinus helleri; $\mathrm{Pl}$, Platyrrhinus lineatus; S1, Sturnira lilium; A1, Estação Ecológica Dahma (EED); A2, Parque Estadual do Prosa (PEP)].

2014b; PorfírIo \& BordignON, 2015), o que pode resultar da baixa abundância de capturas dessa espécie em ambas as áreas amostradas. Embora tenha sido registrado somente o consumo 
de $P$. tuberculatum, a análise de uma única amostra fecal não representa a dieta real da espécie. De forma complementar a análise das fezes, a captura de um indivíduo carregando um fruto de Ficus insipida demonstra a possível utilização desse item por A. planirostris, como relatado por MARTINS et al. (2014b) em Campo Grande.

A similaridade na dieta das populações de $A$. lituratus da EED e do PEP foi elevada $(\mathrm{Cmh}=0,7894)$, porém a similaridade entre a dieta da população de $A$. planirostris com ambas as populações de $A$. lituratus, sendo de $\mathrm{Cmh}=0,4000$ com a população da EED foi de $\mathrm{Cmh}=0,5217 \mathrm{com}$ a do PEP. A similaridade de dieta entre as espécies congêneres de Artibeus é menor do que o esperado, sendo potencialmente influenciada pela pequena quantidade de amostras obtidas para A. planirostris. A análise de mais amostras fecais de A. planirostris possivelmente demonstraria a utilização de outros itens e uma dieta mais diversa, resultando em maior similaridade com a dieta de $A$. lituratus, como verificado por MARTINS et al. (2014a), que relataram $66 \%$ de similaridade de dieta entre essas espécies.

A dieta de $S$. lilium da EED revelou maior similaridade com a população de A. lituratus do $\mathrm{PEP}(\mathrm{Cmh}=0,8571)$, enquanto a dieta da população de $S$. lilium do PEP foi mais similar à de $C$. perspicillata da EED $(\mathrm{Cmh}=0,9974)$. Essas relações de similaridade foram determinadas pelo predomínio dos mesmos itens alimentares nas dietas das populações relacionadas. Embora a dieta de S. lilium seja frequentemente relatada como predominante em frutos de Solanum (e.g. Aguirre et al., 2003; PAssos et al., 2003; BRito et al., 2010), é possível a utilização em frequência elevada de frutos de P. tuberculatum (Munin et al., 2012), registrado aqui como um importante recurso para a espécie.

Os morcegos do gênero Platyrrhinus são predominantemente frugívoros, mas podem incluir em sua dieta insetos, néctar, pólen e folhas (WILLIG \& Hollander, 1987), característica essa apresentada por $P$. lineatus em estudos desenvolvidos no Mato Grosso do Sul (e.g. Munin et al., 2012; MARTINS et al., 2014b; PORFírIO \& BORDIGNON, 2015). Embora tenham sido obtidas poucas amostras fecais de $P$. lineatus e $P$. helleri, os dados aqui apresentados complementam os resultados de MArTins et al. (2014a) em Campo Grande, acrescentando P. tuberculatum a lista de itens consumidos por essas espécies. Além disso, o registro do consumo de $F$. insipida por $P$. lineatus no município (MARTINs et al., 2014a) indica uma dieta mais diversa do que a encontrada.

Glossophaga soricina é conhecida por seu hábito nectarívoro, mas pode utilizar frutos como complementação de sua dieta (FABIÁn et al., 2008). A única amostra obtida indica a inclusão de frutos em sua dieta, que já foi relatada consumindo espécies de Moraceae e Piperaceae (Munin et al., 2012; MarTins et al., 2014a) no Mato Grosso do Sul, embora não seja possível inferir a frequência de utilização destes itens.

Embora a análise de agrupamento demonstre dietas baseadas em preferências específicas, o baixo número de amostras para algumas espécies dificulta a caracterização de suas dietas, podendo interferir na organização dos agrupamentos. Um exemplo disso é o agrupamento baseado no consumo de $P$. tuberculatum, que aproximou populações de $A$. planirostris, $P$. helleri e S.lilium que apresentaram esse item na única amostra obtida com as populações de C. perspicillata, que são especialistas no consumo de Piperaceae (BIzERRIL \& RAW, 1997). O grupo de populações consumidoras de Solanum sp. corrobora com o conhecido para a dieta das espécies, pois $S$. lilium é conhecida como especialista em Solanum spp. (AguirRe et al., 2003) e $A$. lituratus é generalista e frequentemente relatada consumindo frutos de Solanaceae (e.g. Mikich, 2002; BrusCo \& TozATO, 2009; Novaes \& Nobre, 2009).

Os resultados obtidos em ambas as unidades de conservação indicam o potencial dos morcegos para a manutenção desses ambientes, exercendo uma importante função na dispersão de sementes. As espécies vegetais consumidas pelos morcegos filostomídeos em ambas as áreas estudadas são pioneiras, apresentando um crescimento influenciado por alterações na estrutura da vegetação ou por presença de bordas e clareiras (LAURANCE et al., 2002), sendo sua dispersão por morcegos essencial para a sucessão e colonização (GALINDO-GoNZÁLEZ, 1998).

$\mathrm{O}$ conhecimento das interações em fragmentos urbanos é de extrema importância para a determinação de seus planos de manejo, em especial por se tratarem de unidades de conservação inseridas em uma matriz urbana. Essas informações permitem o reconhecimento de espécies vegetais atrativas à fauna, principalmente de grupos potencialmente dispersores e capazes de promover a movimentação de sementes entre diferentes remanescentes, garantindo a manutenção dos processos ecológicos e minimizando a simplificação dos sistemas naturais.

Agradecimentos. Ao Programa Institucional de Bolsas de Iniciação Científica PIBIC UCDB/CNPq, pelo financiamento do projeto. Ao SISBIO/ICMBio/MMA pela concessão das licenças. Ao IMASUL e SEMADUR pela permissão de coleta nas áreas de estudo. Ao Dr. Fernando Henrique Martin Gonçalves, pelo auxílio na identificação das sementes e contribuições ao trabalho. A Dra. Carolina Ferreira Santos, pelas contribuições ao trabalho. A Driele Karen Ferreira Soares e Mariana Pires Veiga Martins pelo auxílio nas atividades de campo. E.A.C. dos A. é bolsista CAPES, Processo no $1218-13-1$.

\section{REFERÊNCIAS BIBLIOGRÁFICAS}

Aguiar, L. M. S. \& Marinho-Filho, J. 2007. Bat frugivory in a remnant of southeastern Brazilian atlantic forest. Acta Chiropterologica 9:251260.

Aguirre, L. F.; Herrel, A.; van Damme, R. \& Matthysen, E. 2003. The implications of food hardness for diet in bats. Functional Ecology 17:201-212.

Bernard, E.; Aguiar, L. M. S.; Brito, D.; Cruz-Neto, A. P.; Gregorin, R.; Machado, R. B.; Oprea, M.; Paglia, A. P. \& Tavares, V. C. 2012. Uma análise de horizontes sobre a conservação de morcegos no Brasil. In: Freitas, T. R. O. \& Viera. E. M. eds. Mamíferos do Brasil: genética, sistemática, ecologia e conservação. 2ed. Rio de Janeiro, Sociedade Brasileira de Mastozoologia, p. 19-35.

BizerriL, M. X. A. \& RAw, A. 1997. Feeding specialization of two species of bats and the fruit quality of Piper arboreum in a Central Brazilian gallery forest. Revista de Biologia Tropical 45:913-918.

BREDT, A. \& UIEDA, W. 1996. Bats from urban and rural environments of the Distrito Federal, mid-western Brazil. Chiroptera Neotropical 2:54-57.

Brito, J. E. C.; Gazarini, J. \& ZawadZKi, C. H. 2010. Abundância de frugivoría de quiropterofauna (Mammalia, Chiroptera) de um fragmento 
no noroeste do Estado do Paraná, Brasil. Acta Scientiarium, Biological Sciences 32:265-271.

Brusco, A. R. \& Tozato, H. C. 2009. Frugivoria na dieta de Artibeus lituratus Olfers, 1818 (Chiroptera, Phyllostomidae) no Parque do Ingá, Maringá/PR. Revista F@pciência 3:19-29.

Charles-Dominique, P. 1986. Inter-relations between frugivorous vertebrates and pioneer plants: Cecropia, birds and bats in French Guiana. In: Estrada, A. \& Fleming, T. H. eds. Frugivores and seed dispersal., Dordrecht, Junk Publishers, p. 119-136.

Deminicis, B. B.; Vieira, H. D; AraúJo, S. A. C; Pádua, F. T. \& Chambela Neto, A. 2009. Dispersão natural de sementes: importância, classificação e sua dinâmica nas pastagens tropicais. Archivos de Zootecnia 58:3558.

FABiÁn, M. E.; RuI, A. M. \& WAEChTER, J. L. 2008. Plantas utilizadas como alimento por morcegos (Chiroptera, Phyllostomidae), no Brasil. In: Reis, N. R.; Peracchi, A. L. \& Santos, G. A. S. D. eds. Ecologia de morcegos. Londrina, Technical Books Editora, p. 51-70.

FeIBER, S. D. 2004. Áreas verdes urbanas imagem e uso: o caso do passeio público de Curitiba, PR. RA'E GA 8:93-105.

Fenton, M. B.; Acharya, L.; Audet, D.; Hickey, M. B. C.; Merriman, C.; OвRISt, M. K. \& Syme, D. M. 1992. Phyllostomid bats (Chiroptera: Phyllostomidae) as indicators of habitat disruption in the Neotropics. Biotropica 24:440-446.

Fleming, T. H. \& SoSA, V. J. 1994. Effects of nectarivorous and furgivorous mammals on reproduce sucess of seeds. Journal of Mammalogy 75:845-851.

GaLindo-GonzÁLEZ, J. 1998. Dispersión de semillas por murciélagos: su importancia em la conservación y regeneración del bosque tropical. Acta Zoologica Mexicana 73:57-74.

Galindo-GonzÁlez, J.; Guevara, S. \& Sosa, V. J. 2000. Bat and bird generated seed rains at isolate trees in pastures in a tropical rainforest. Conservation Biology 14(6): 1693-1703.

Giannini, N. P. \& KalKo, E. K. V. 2004. Trophic structure in a large assemblaje of phyllostomid bats in Panama. Oikos 105:209-220.

Hammer, O.; Harper, D. A. T. \& Rian, P. D. 2001. Past: Palaeonthological statistics software package for education and data analysis. Paleontologia Electronica 4(1):1-9.

Honer, M. R. 1993. Precipitação pluviométrica na região de Campo Grande, MS: uma análise do período 1970-1991. Campo Grande, EMBRAPA-CNPGC. 46p.

Hutson, A. M.; Mickleburgh, S. P. \& Racey, P. A. 2001. Microchiropteran bats: global status survey and conservation action plan. Gland, Switzerland and Cambridge, IUCN/SSC Chiroptera Specialist Group, 258p.

Kageyama, P. \& Gandara, F. B. 2003. Restauração e conservação de ecossistemas tropicais. In: CUllen JúNIOR, L.; Valladares-PÁdua, C. \& RUDRAN, R. orgs. Métodos de estudos em biologia da conservação e manejo da vida silvestre. Curitiba, UFPR/Fundação o Boticário de Proteção à Natureza, p. 383-394.

Laurance, W. F.; Lovejoy, T. E.; Vasconcelos, H. L.; Bruna, E. M.; Didham, R. K.; Stouffer, P. C.; Gascon, C.; BierregaArd, R. O.; Laurance, S. G. \& Sampaio, E. 2002. Ecosystem decay of Amazonian Forest fragments: a 22-year investigation. Conservation Biology 16(3):605-618.

Le Bourlegat, C. A. 2003. A fragmentação da vegetação natural e o paradigma do desenvolvimento rural. In: CosTA, R. B. ed. Fragmentação Florestal e alternativas de desenvolvimento rural na Região CentroOeste. Campo Grande, Universidade Católica Dom Bosco, p. 1-24.

Lima, I. P. 2008. Espécies de morcegos (Mammalia, Chiroptera) registradas em parques nas áreas urbanas do Brasil e suas implicações no uso deste ambiente. In: Reis, N. R.; Peracchi, A. L. \& Santos, G. A. S. D. eds. Ecologia de Morcegos. Londrina, Technnical Books, p. 71-85.

Martins, M. P. V.; Torres, J. M. \& ANJos, E. A. C. 2014a. Dieta de morcegos filostomídeos (Mammalia, Chiroptera, Phyllostomidae) em fragmento urbano do Instituto São Vicente, Campo Grande, Mato Grosso do Sul. Papéis Avulsos de Zoologia 54:299-305.

Martins, M. P. V.; Torres, J. M. \& Anjos, E. A. C. 2014b. Dieta de morcegos frugívoros em remanescente de Cerrado em Bandeirantes, Mato Grosso do Sul. Biotemas 27:129-135.

McAleece, N.; Lambshead, P. J. D.; Paterson, G. L. J. \& Gage, J. G. 1997. Biodiversity professional. Beta-Version. The Natural History Museum $\&$ the Scottish Association for Marine Sciences. Disponível em $<\mathrm{http}: / /$ biodiversity-pro.software.informer.com/2.0/>. Acesso em 30.10.2016.
Melo, A. G. C.; Carvalho, D. A.; Castro, G. C. \& Machado, E. L. M. 2011. Fragmentos florestais urbanos. Revista Científica Eletrônica de Engenharia Florestal 17(1):58-79.

Mikich, S. B. 2002. A dieta de morcegos frugívoros (Mammalia, Chiroptera, Phyllostomidae) de um pequeno remanescente de Floresta Estacional Semidecidual do sul do Brasil. Revista Brasileira de Zoologia 19(1):239-249.

Mikich, S. B.; Bianconi, G. V.; Parolin, L. C. \& Almeida, A. 2015. Serviços ambientais prestados por morcegos frugívoros na recuperação de áreas degradadas. In: Parron, L. M.; Garcia, J. R.; Oliveira, E. B.; Brown, G. G. \& Prado, R. B. orgs. Serviços ambientais em sistemas agrícolas e florestais do Bioma Mata Atlântica. Brasília, Embrapa, p. 248-256.

Moffatt, S. F.; McLachlan, S. M. \& KenKel, N. C. 2004. Impacts of land use on riparian forest along an urban-rural gradient in southern Manitoba. Plant Ecology 174:119-135.

MoRISITA, M. 1959. Measuring of interspecific association and similarity between communities. Memoirs of the Faculty of Science, Kyushu University, Series E. Biology 3:65-80.

Munin, R. L.; Fischer, E. \& GonÇALVES, F. 2012. Food habits and dietary overlap in a Phyllostomid Bat Assemblage in the Pantanal of Brazil. Acta Chiropterologica 14(1):195.204.

Novaes, R. L. M. \& Nobre, C. C. 2009. Dieta de Artibeus lituratus (Olfers, 1818) em área urbana da cidade do Rio de Janeiro: frugivoria e novo registro de folivoria. Chiroptera Neotropical 15(2):487-493.

Oliveira, A. K. M. \& Lemes, F. T. F. 2010. Artibeus planirostris como dispersor e indutor de germinação em uma área do Pantanal do Negro, Mato Grosso do Sul, Brasil. Revista Brasileira de Biociências 8:49-52.

Passos, F. C. \& Graciolli, G. 2004. Observações da dieta de Artibeus lituratus (Olfers) (Chiroptera, Phyllostyomidae) em duas áreas do sul do Brasil. Revista Brasileira de Zoologia 21:487-489.

Passos, F. C.; Silva, W. R.; Pedro, W. A. \& Bonin, M. R. 2003. Frugivoria em morcegos (Mammalia, Chiroptera) no Parque Estadual Intervales, sudeste do Brasil. Revista Brasileira de Zoologia 20:511-517.

Peel, M. C.; Finlayson, B. L. \& McMahon, T. A. 2007. Update world map of the Koppen-Geiger climate classification. Hidrolody and Earth System Sciences 11:1633-1644.

Pinto, D. \& Ortêncio Filho, H. 2006. Dieta de quatro espécies de filostomídeos frugívoros (Chiroptera, Mammalia) do Parque Nacional do Cinturão Verde de Cianorte, Paraná, Brasil. Chiroptera Neotropical 12(2):274-279.

Porfírio, G. \& Bordignon, M. O. 2015. Phyllostomid bats and their diets at Urucum Massif, Mato Grosso do Sul, Brazil. Chiroptera Neotropical 21(2):1332-1337.

Rares, C. S. \& Brandimarte, A. L. 2014. O desafio da conservação de ambientes aquáticos e manutenção de serviços ambientais em áreas verdes urbanas: o caso do Parque Estadual da Cantareira. Ambiente \& Sociedade 17(2):111-128.

Reis, N. R.; Peracchi, A. L; Pedro, W. A. \& Lima, I. P. 2007. Morcegos do Brasil. Londrina, Universidade Estadual de Londrina. 253p.

RickLEFs, R. E. 1990. Ecology. New York, W. H. Freeman. 896p.

Sato, T. M.; Passos, F. C. \& Nogueira, A. C. 2008. Frugivoria de morcegos (Mammalia, Chiroptera) em Cecropia pachystachya (Urticaceae) e seus efeitos na germinação de sementes. Papéis Avulsos de Zoologia 48:19-26.

Silva, J. M. C. \& Tabarelli, M. 2000. Tree species impoverishment and the future flora of the Atlantic forest of northeast Brazil. Nature 404(6773): 72-74.

Straube, F. C. \& Bianconi, G. V. 2002. Sobre a grandeza e a unidade utilizada para estimar esforco de captura com utilização de redes-deneblina. Chiroptera Neotropical 8:150-152.

TeIXeIra, R. C.; CorrêA, C. E. \& Fischer, E. 2009. Frugivory by Artibeus jamaicensis (Phyllostomidae) bats in the Pantanal, Brazil. Studies on Neotropical Fauna and Environmental 44:7-15.

Vizotto, L. D. \& Taddei, V. A. 1973. Chave para a identificação de quirópteros brasileiros. Boletim de Ciências, Faculdade de Filosofia, Ciências e Letras 1:1-72.

WiLlig, M. R. \& Hollander, R. R. 1987. Vampyrops lineatus. Mammalian Species 275:1-4.

WILLSON, M. F. 1991. Dispersal of seeds by frugivorous animals in temperate forests. Revista Chilena de Historia Natural 64:537-554.

ZortÉA, M. 2007. Subfamília Stenodermatinae. In: ReIs, N. R.; PerACCHI, A. L.; Pedro W. A. \& Lima, I. P. eds. Morcegos do Brasil. Londrina, Universidade Estadual de Londrina, p. 107-128. 